\title{
Development of advanced fragrant rice lines from MR269 $\times$ Basmati 370 through marker-assisted backcrossing
}

\begin{abstract}
Fragrance in rice is an appealing attribute to consumers. The increasing demand for fragrant rice highlights the need to develop fragrant rice variety that suit the preference of local consumers in addition to reduce fragrant rice imports. Marker-assisted backcrossing (MABC) was employed to develop advanced fragrant rice lines from the cross between MR269 and Basmati 370. MR269 is a Malaysian high-yielding rice variety but non-fragrant and was used as recurrent parent whereas Basmati 370 is a well-known fragrant traditional rice variety and was used as donor parent for the fragrance gene. Two generations of backcrosses and a generation of selfing were conducted to introgress the fragrance gene and restore the recurrent parent genome in the backcross progenies. As a result, 14 advanced fragrant rice lines were developed. These advanced fragrant rice lines carried homozygous alleles for the fragrance gene, similar to Basmati 370 . The average recovery of recurrent parent genome was 88.4\%. Besides being fragrant, the advanced fragrant rice lines also had most of the morphological and agronomical traits similar to MR269. Grain quality of the advanced fragrant rice lines in terms of gelatinization temperature, amylose content and gel consistency are also similar to both parents. Besides, the advanced fragrant rice lines had 2-acetyl-1pyrroline content similar to Basmati 370. MABC approach applied in this study has successfully introgressed the fragrance gene and accelerated the recovery of recurrent parent genome in advanced fragrant rice lines, therefore these lines can be delivered to the farmers and consumers for use in due time.
\end{abstract}

Keyword: Fragrance; Foreground selection; Background selection; Recurrent parent genome recovery; Marker-assisted backcrossing 